\title{
PRONÓSTICO DE LA FLUCTUACIÓN POBLACIONAL DEL MINADOR DE LA HOJA DE CRISANTEMO LIRIOMYZA HUIDOBRENSIS BLANCHARD (DIPTERA: AGROMYZIDAE) MEDIANTE MODELOS DE SERIES DE TIEMPO
}

\author{
Evelia Hernández Regalado', Jorge Vera Graziano', \\ Gustavo Ramírez VAlverde ${ }^{2}$, Sergio PÉrez Elizalde², \\ José López Collado ${ }^{3}$, Néstor BaUtista Martínez ${ }^{1}$, Victor M. Pinto ${ }^{4}$ \\ 1Instituto de Fitosanidad, Entomología y Acarología, Colegio de Postgraduados, Campus Montecillo, \\ Carr. México-Texcoco, km 36.5 C. P. 56230 Edo. de Mex. MÉXICO (regalado@colpos.mx), \\ (graziano@colpos.mx), (nestor@colpos.mx) \\ 2Instituto de Socioeconomia, Estadística e Informática, Estadística. Colegio de Postgraduados. \\ Campus Montecillo, Carr. México-Texcoco, km 36.5 C. P. 56230 Edo. de Mex. MÉXICO \\ (gramirez@colpos.mx), (sergiop@colpos.mx) \\ 3Instituto de Fitosanidad, Entomología y Acarología, Colegio de Postgraduados, Campus Veracruz, \\ Carr. Xalapa-Veracruz, km. 88.5 Apdo. Postal 421 C.P. 1700 Veracruz. MÉXICO \\ (jlopez@colpos.mx). \\ 4Programa de Posgrado en Protección Vegetal, Depto. de Parasitología Agrícola, Universidad Autó- \\ noma Chapingo, Carr. México-Texcoco, km 38.5 C. P. 56230 Edo. de Mex. MÉXICO \\ (vmpinto@correo.chapingo.mx)
}

Hernández Regalado, E., J. Vera Graziano, G. Ramírez Valverde, S. Pérez Elizalde, J. López Collado, N. Bautista Martínez y V. M. Pinto. 2009. Pronóstico de la fluctuación poblacional del minador de la hoja de crisantemo Liriomyza huidobrensis Blanchard (Diptera: Agromyzidae), mediante modelos de series de tiempo. Acta Zool. Mex. (n. s.) 25(1): 21-32

RESUMEN. El presente trabajo se realizó con el propósito de modelar la fluctuación poblacional del minador de la hoja de crisantemo Liriomyza huidobrensis (Blanchard) a fin de encontrar modelos de predicción con el método de Box \& Jenkins que pudieran representar y predecir la densidad de población del insecto en su estado de larva. Se trabajó en dos ciclos de cultivo con duración de cuatro meses cada uno obteniéndose dos poblaciones de insectos. El número de insectos se registró periódicamente cada dos días obteniendo 61 observaciones para cada ciclo de cultivo; por fecha de lectura se anotó el número de larvas vivas; teniendo así dos series de tiempo. Las primeras 55 observaciones de cada serie se analizaron para la obtención del modelo de acuerdo a la metodología de Box \& Jenkins y las seis observaciones finales ayudaron a validar la capacidad de predicción del modelo encontrado. En el proceso de identificación del modelo para la representación de cada una de las series observadas se probaron transformaciones de éstas, siendo los ajustes más adecuados para la serie 1 la transformación con raíz cuadrada, y para la serie 2 la transformación de Box-Cox con potencia (0.387455). En ambas series las autocorrelaciones (FAC) denotaron estacionaridad y las autocorrelaciones parciales (FACP) se interrumpieron en la autocorrelación 1. El modelo estimado para 
la serie 1 fue $\mathrm{Y}_{\mathrm{t}}=0.246842+0.978041 \mathrm{Yt}-1$ y para la serie 2 fue $\mathrm{Y}_{\mathrm{t}}=0.283874+0.985939 \mathrm{Yt}-1$. La verificación del modelo ajustó bien los datos al obtener ruido blanco en los residuales de la FAC y FACP de los modelos estimados. Se generaron dos modelos estacionarios de series de tiempo autorregresivos del tipo AR (1) que representaron a las series observadas de L. huidobrensis, ajustandose bien al comportamiento real de sus poblaciones y logrando predecir satisfactoriamente valores de la fluctuación poblacional del insecto.

Palabras clave: Liriomyza huidobrensis, modelos matemáticos, ARIMA, predicción de plagas.

Hernández Regalado, E., J. Vera Graziano, G. Ramírez Valverde, S. Pérez Elizalde, J. López Collado, N. Bautista Martínez \& V. M. Pinto. 2009. Prediction of the population fluctuation of the Chrysanthemum leaf miner Liriomyza huidobrensis Blanchard (Diptera: Agromyzidae), using time series models. Acta Zool. Mex. (n. s.) 25(1): 21-32.

ABSTRACT. The present study had the objective of modelling population fluctuation of chrysanthemum leaf miner (Liriomyza huidobrensis Blanchard), using the Box \& Jenkins method, in order to find prediction models, which could represent and adequately predict population density of the insect at its larval stage. The work was carried out in two four-month crop cycles. The number of insects was recorded periodically every two days, resulting in 61 observations for each crop cycle. The number of live larvae was registered by reading date, obtaining two time series. The first 55 observations of each series were analyzed to set the model according to the Box \& Jenkins' method, and the six final observations helped to validate the prediction capacity of the model found. In the process of identifying the model for the representation of each of the observed series, their transformation was tested, finding for series 1 that the transformation with square root had the most adequate fit, and for series 2 , the transformation of Box-Cox with power $(0.387455)$ was the most adequate. In both series, the autocorrelations (FAC) showed stationarity, and partial autocorrelations (FACP) were interrupted in autocorrelation 1. The estimated model for series 1 was $\mathrm{Yt}=0.246842+0.978041 \mathrm{Yt}-1$, and for series 2 , it was $\mathrm{Yt}=0.283874+0.985939 \mathrm{Yt}-1$. The testing of the model fitted the data well, obtaining white noise in the residuals of FAC and FACP of the estimated models. Two stationary models of autoregressive time series of the AR (1) type were generated, representing the observed series of $L$. huidobrensis, well fitting the true behaviour of their populations and achieving to satisfactorily forecast future values of the insect population fluctuation.

Key words: Liriomyza huidobrensis, mathematical models, ARIMA, pest prediction.

\section{INTRODUCCIÓN}

En México se siembran aproximadamente 2300 ha de crisantemo Dendranthema grandiflora Tzvelev (Anuario Estadístico de la Producción Agrícola 2005). Una de las plagas primarias más importante de este cultivo es el complejo de "minadores de la hoja" (Parrella et al. 1981; Parrella 1987). En la región productora de crisantemo de Texcoco, Estado de México, se han documentado tres especies de minadores: Liriomyza trifolii Burgess, L. sativa Blanchard y L. huidobrensis Blanchard; siendo esta última la dominante (Huerta 2000). Las galerías que las larvas ocasionan en las hojas afectan la capacidad fotosintética de la planta y reducen el valor comercial de las flores (Foster \& Sánchez 1988; Perrella et al. 1981). El control de L. huidobrensis se complica por falta de estudios que pronostiquen su dinámica poblacional y que permitan identificar los factores que influyen en los cambios en la densidad de su población (Chandler \& Thomas 1983; Harvir Singh et al. 1996). Existe un grupo de 
modelos estocásticos conocidos como series de tiempo desarrollados por Box \& Jenkins (1976) que permiten una predicción rápida de series biológicas. Infante et al. (1983) destacan las ventajas del método de Box y Jenkins para un adecuado pronóstico de poblaciones biológicas. Los modelos de series de tiempo se han empleado para el estudio y predicción de fenómenos físicos, biológicos, así como en economía, geofísica, demografía (Gottman 1981; Scargle 1982; Weigend \& Gershenfeld 1994; Jones \& Hulme 1996; Zeger et al. 2000). También se han usado en otros trabajos como los de Infante et al. (1983), con observaciones semanales del díptero Oxisarcodexia ventricosa, realizando un análisis de series de tiempo con modelos autorregresivos integrados de promedios móviles (ARIMA) obteniendo satisfactoriamente modelos que predijeron las densidades del insecto. Pinto et al. (1990) se basaron en el análisis de series de tiempo empleando el método de Box y Jenkins para describir y predecir el comportamiento de dos poblaciones de Zabrotes subfasciatus (Coleoptera: Bruchidae) en condiciones de laboratorio, obteniendo dos modelos, ambos de tipo estacional. Williams \& Liebhold (1995), mediante un análisis de series de tiempo (de información recabada durante 55 años), determinaron dos procesos autorregresivos de 1 er y 2 do orden que evidenciaron la sincronización de la emergencia del lepidóptero Lymantria dispar $\mathrm{L}$ en tres estados de Estados Unidos de Norteamérica. Redfern \& Hunter (2005) desarrollaron cuatro modelos de predicción mediante series de tiempo al monitorear cada año desde 1966 al 2002 dos poblaciones de Taxomyia taxi (Cecidomyiidae) y de dos especies de parasitoides Mesopolobus diffinis y Torymus nigritarsus, detectando que el comportamiento de las poblaciones se repite en ciclos de 14 años, y que los parasitoides y el huésped regulan su población por ser denso-dependientes.

El objetivo de la presente investigación consistió en generar un modelo de predicción de la densidad de población de larvas minadoras del crisantemo Liriomyza huidobrensis Blanchard mediante el método de Box y Jenkins.

\section{MATERIAL Y MÉTODOS}

Lugar de estudio. La toma de datos de la fluctuación poblacional en campo se llevó a cabo en las instalaciones de la empresa MICROPLAS en la Col. Netzahualcóyotl, municipio de Texcoco, Estado de México, ubicado geográficamente a $2241 \mathrm{msnm}$ en las coordenadas $19^{\circ} 30^{\prime} \mathrm{N}$ y $98^{\circ} 51^{\prime} \mathrm{O}$; con clima $\mathrm{Cb}(\mathrm{w} 1)(\mathrm{w})\left(\mathrm{i}\right.$ ')g; temperatura media anual de $15.9^{\circ} \mathrm{C}$ y precipitación pluvial media anual de $710.3 \mathrm{~mm}$ (García 1987).

Periodo experimental. Se trabajó en dos ciclos de cultivo con duración de cuatro meses cada uno, en los cuales se trasplantaron 3,600 plántulas de crisantemo de la variedad "diamante blanco" (flor tipo pompón), libres de plagas y enfermedades, bajo superficie de invernadero de $180 \mathrm{~m}^{2}$. El primer ciclo trasplantado el 22 de 
septiembre de 2006 y el segundo ciclo trasplantado el 22 de enero del 2007. El manejo del cultivo no se hicieron aplicaciones de de insecticidas.

Muestreo. De una población de 3,600 plantas de crisantemo se realizó un muestreo sistemático de 1 en 20 (Castillo, 2005) y se obtuvo una muestra al azar de cinco hojas en el tercio medio de la planta, de la cuales se registró el número de minas con larvas vivas, comúnmente presentes en esa parte de la planta.

Fluctuación poblacional de Liriomyza huidobrensis. La lectura de la población se hizo cada dos días de manera hasta completar un total de 61 observaciones para cada ciclo del cultivo. Por fecha de lectura se anotó el número de larvas vivas por hoja en su probable desarrollo larval L3 y L4, obteniéndose dos series de tiempo.

Análisis de datos. La información recabada de los muestreos de larvas de Liriomyza huidobrensis a través del tiempo (23 septiembre de 2006 a 21 de enero de 2007 y del 23 de enero al 23 de mayo de 2007) se trabajó con modelos de series de tiempo utilizando el método de Box \& Jenkins (1976), tomando para cada serie las primeras 55 observaciones de ésta para el análisis de los datos, conservándose las últimas 6 observaciones para determinar la capacidad de predicción del modelo encontrado y validar su eficacia. Los modelos de series de tiempo de L. huidobrensis se obtuvieron con el programa Statgraphics 5.1 (Statgraphics, 2001)

\section{RESULTADOS}

Serie 1. La Figura 1 muestra la serie de tiempo obtenida para la serie 1 en donde se detecta bajo número de especímenes en las observaciones 1 a la 36 . La parte complementaria de la serie muestra una clara tendencia ascendente a partir de las observaciones 37 a 55 .

Identificación del modelo. La función de autocorrelación (FAC) de esta serie (Figura 2) tiene caída exponencial que da evidencia de la estacionariedad de la serie. La FACP (Figura 3) se interrumpe en la autocorrelación parcial 1 y conjuntamente con el comportamiento de la FAC sugiere que los datos se ajustan a un modelo AR (1).

En el proceso de identificación del modelo se revisó su ajuste y sus supuestos, encontrando que el ajuste más adecuado fue la transformación con raíz cuadrada de los datos.

Estimación de los parámetros del modelo. El modelo que mejor ajustó la serie 1 fue un modelo autorregresivo de orden 1 que se expresa como:

Donde:

$$
\mathrm{Yt}=\delta+\emptyset_{1} \mathrm{Y}_{\mathrm{t}-1}+\mathrm{a}_{\mathrm{t}}
$$

$\mathrm{Yt}=$ pronóstico de la serie de tiempo (valor de la serie en el tiempo $\mathrm{t}$ )

$\delta=$ constante del modelo

$\varnothing_{1}=$ coeficiente de autoregresión

$a_{t}=$ error aleatorio 


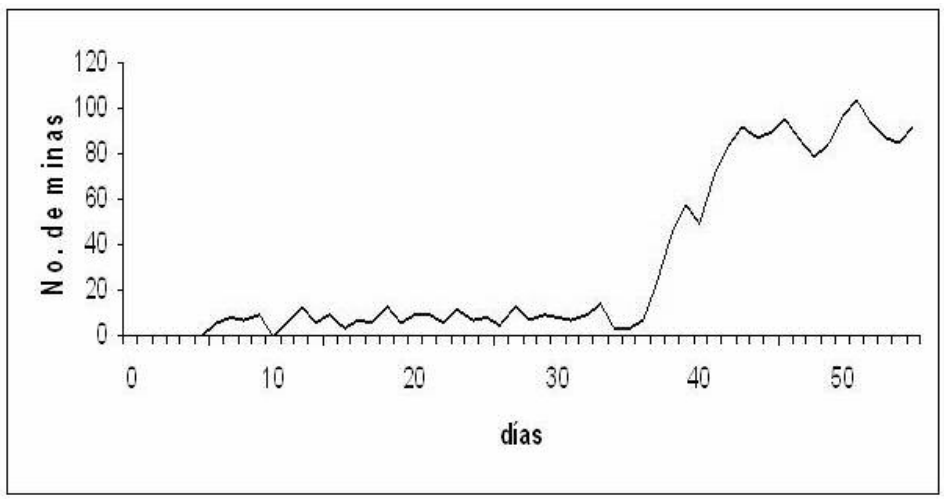

Figura 1. Serie 1 con datos del número de minas de L. huidobrensis observadas en cada fecha de muestreo.

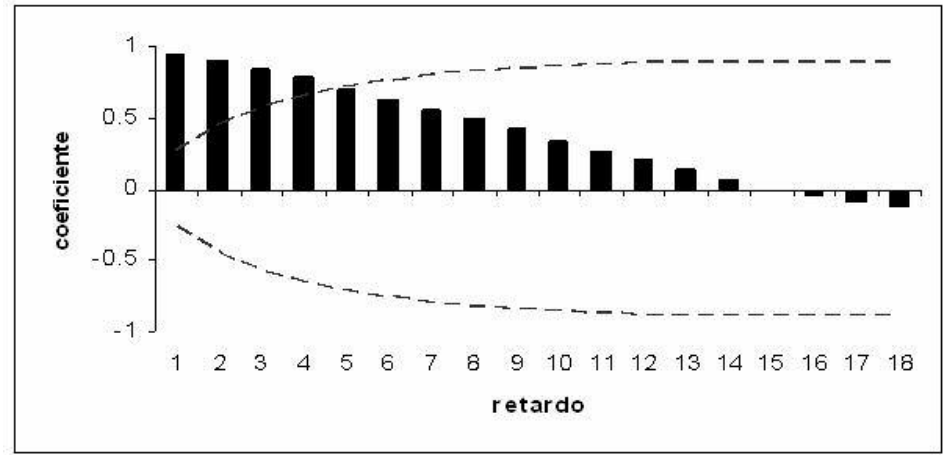

Figura 2. Función de autocorrelación (FAC) de la serie 1 (las líneas punteadas corresponden a \pm dos veces el valor estándar de los coeficientes).

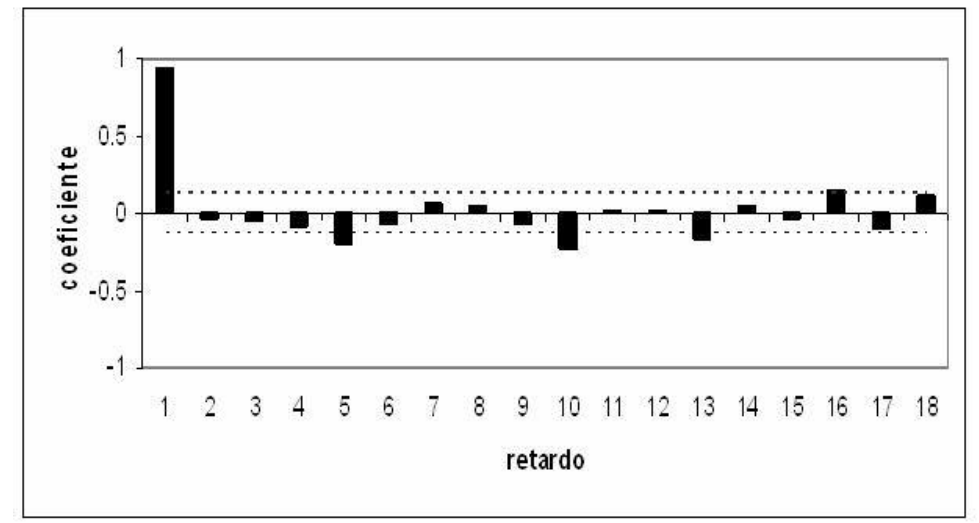

Figura 3. Función de autocorrelación parcial (FACP) de la serie 1 (las líneas punteadas corresponden a \pm dos veces el valor estándar de los coeficientes). 
Hernández et al. Modelo poblacional para Liriomyza huidobrensis

Los valores estimados de los parámetros del modelo se anotan en el Cuadro 1, por lo que el modelo estimado es: $\hat{\mathrm{Y}} \mathrm{t}=0.246842+0.978041 \mathrm{Y}_{\mathrm{t}-1}$

Cuadro 1. Estimadores de los parámetros del modelo AR(1) de la serie 1.

\begin{tabular}{lcccc}
\hline Parámetro & Valor estimado & Error estándar & Valor de T & Valor de P \\
\hline$\varnothing 1$ & 0.978041 & 0.0432154 & 22.6318 & 0.000000 \\
Media & 11.2412 & 12.0905 & 0.929755 & 0.356713 \\
$\delta$ & 0.246842 & & & \\
\hline
\end{tabular}

Verificación del modelo AR (1). Las Figuras 4 y 5 muestran que los residuales son ruido blanco; evidencia de que el modelo escogido ajusta bien a los datos.

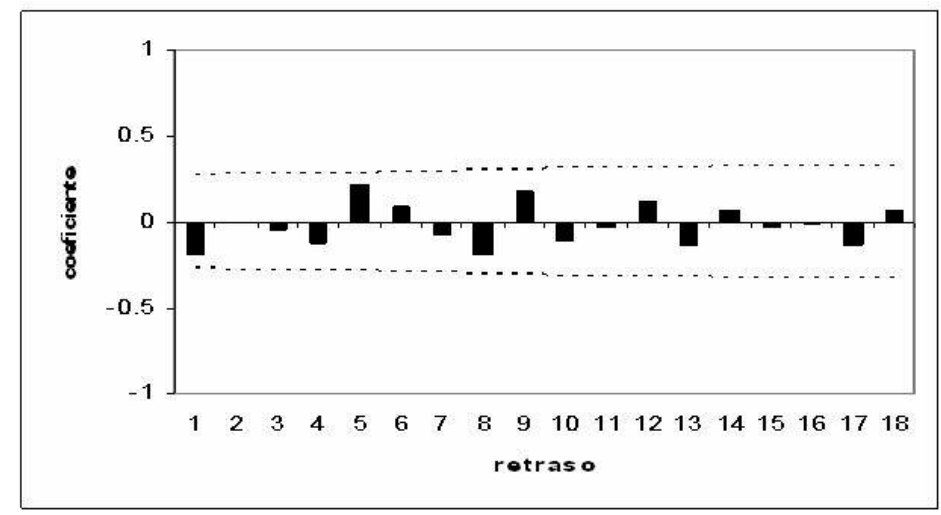

Figura 4. Función de autocorrelación (FAC) de los residuales del modelo AR(1) de la serie 1 (las líneas punteadas corresponden a \pm dos veces el valor estándar de los coeficientes).

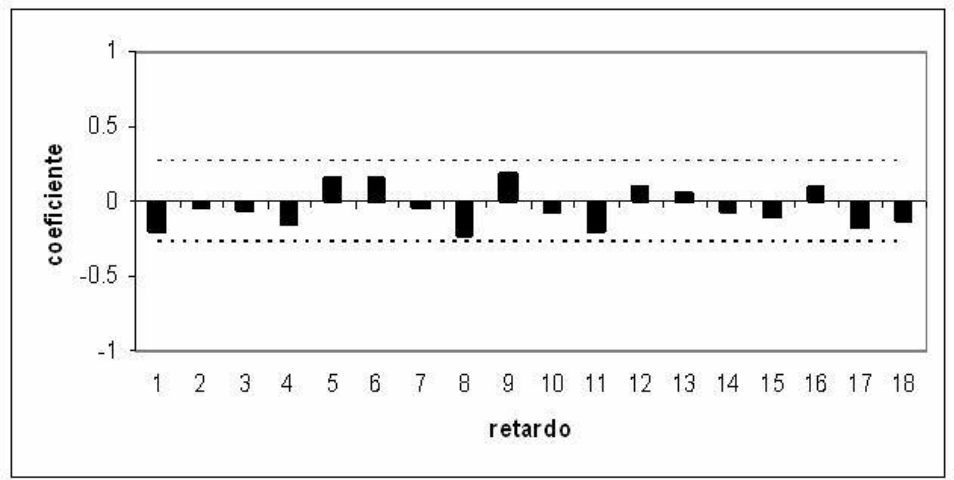

Figura 5. Función de autocorrelación parcial (FACP) de los residuales del modelo AR(1) de la serie 1 (las líneas punteadas corresponden a \pm dos veces el valor estándar de los coeficientes). 
La Figura 6 muestra la capacidad de ajuste del modelo autorregresivo de orden 1 a la serie 1. Puede notarse que el ajuste fue satisfactorio ya que el comportamiento de la serie estimada se apega al comportamiento de las primeras 55 observaciones realizadas sobre el insecto $L$. huidobrensis

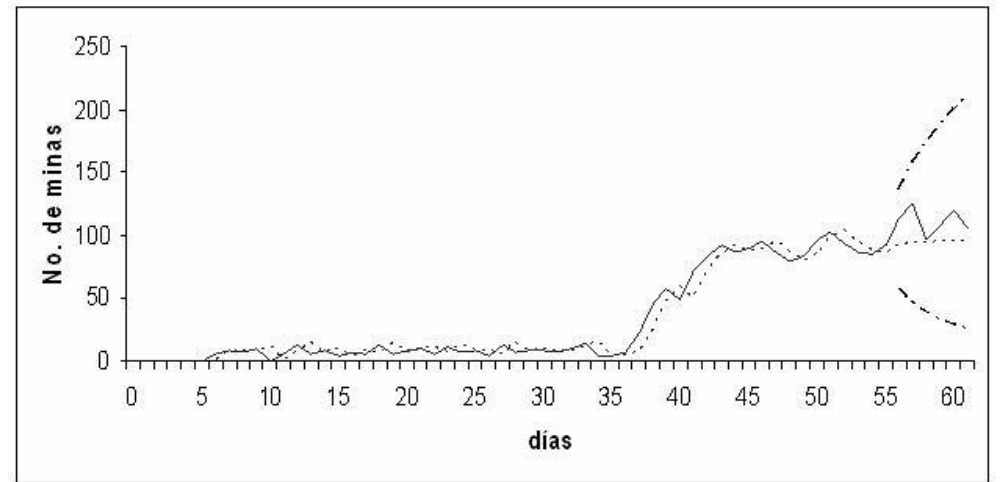

Figura 6. Ajuste del modelo AR(1) a la serie 1 ( _ valores observados, ....valores predichos, _. límites de confianza)

Predicción de la serie 1 con el modelo AR (1). La capacidad de predicción del modelo seleccionado fue confirmada comparando sus resultados con las seis observaciones originales restantes (56-61) de la serie 1 que se conservaron para tal fin (Cuadro 2; Figura 6), donde se comprueba que el modelo obtenido arrojó valores muy cercanos a los originalmente observados en campo.

Cuadro 2. Observaciones finales de la serie 1, su predicción a través del modelo AR(1) y sus límites de confianza al $95 \%$

\begin{tabular}{lcccc}
\hline Período & Serie observada & Serie predicha & Límite inferior & Límite superior \\
\hline 56 & 113 & 92.6962 & 57.4199 & 136.38 \\
56 & 126 & 93.3796 & 46.1777 & 157.031 \\
58 & 97 & 94.0504 & 38.733 & 173.51 \\
59 & 109 & 94.7088 & 33.2137 & 187.705 \\
60 & 120 & 95.355 & 28.8928 & 200.358 \\
61 & 105 & 95.9891 & 25.3973 & 211.855 \\
\hline
\end{tabular}

Serie 2. En la Figura 7 se observa el comportamiento de la población 2 de $L$. huidobrensis, evidenciándose que el número de minas incrementó notablemente a partir de la observación 10 mostrando tener tendencia ascendente de su fluctuación poblacional. 
Hernández et al. Modelo poblacional para Liriomyza huidobrensis

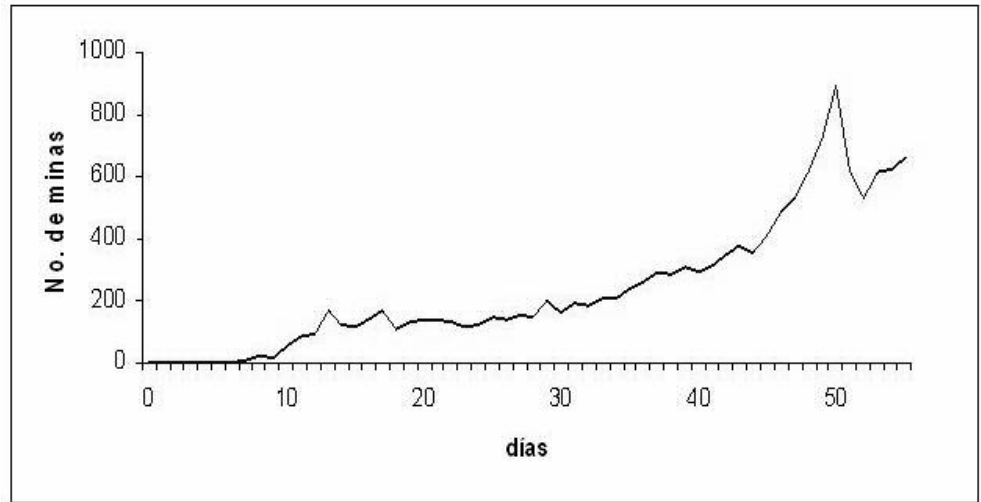

Figura 7. Serie 2 con datos del número de minas de L. huidobrensis observadas en cada fecha de muestreo.

Identificación del modelo. La FAC de la serie 2 (Figura 8) muestra caída exponencial, lo cual denota estacionariedad de la serie. La FACP (Figura 9) interrumpe en la autocorrelación parcial 1 y conjuntamente con el comportamiento de la FAC sugiere que los datos se ajustan a un modelo AR (1). En el proceso de identificación del modelo para la representación de la serie 2 se probaron varias transformaciones de la serie observada, encontrándose que la transformación de BoxCox con potencia $(0.387455)$ fue la más adecuada. Finalmente se eligió el modelo AR (1) con constante.

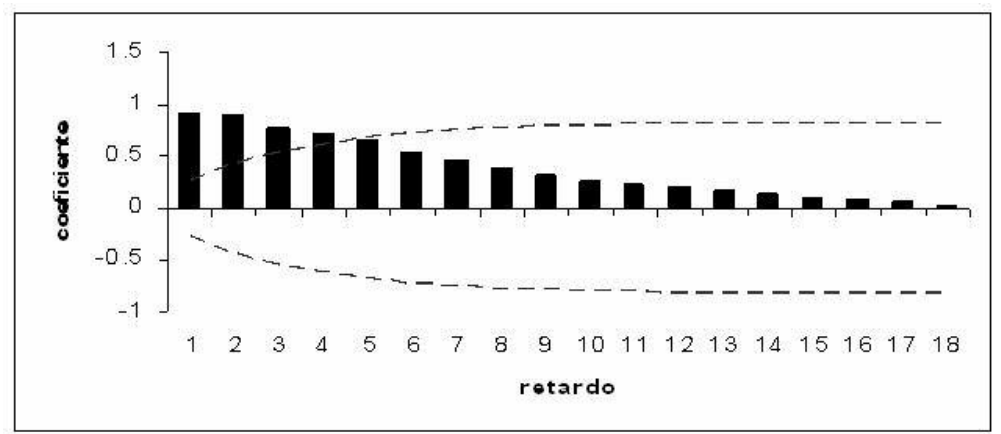

Figura 8. Función de autocorrelación (FAC) de la serie 2 (las líneas punteadas corresponden a \pm dos veces el valor estándar de los coeficientes).

Estimación de los parámetros del modelo. Los valores estimados del modelo se anotan en el Cuadro 3, sustituidos en el modelo propuesto se tiene: $\mathrm{Yt}=0.283874+$ 0.985939 Yt-1 


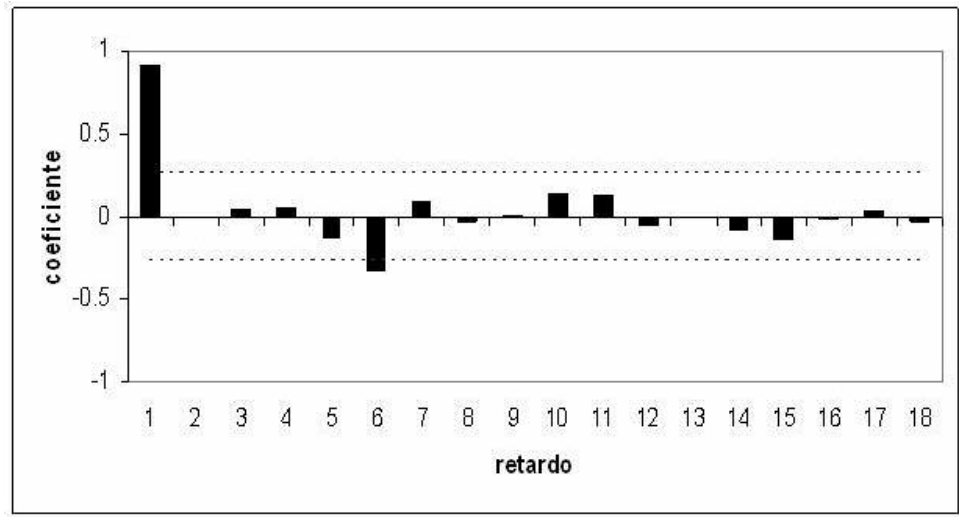

Figura 9. Función de autocorrelación parcial (FACP) de la serie 2 (las líneas punteadas corresponden $\mathrm{a} \pm$ dos veces el valor estándar de los coeficientes).

Cuadro 3. Estimadores de los parámetros del modelo AR(1) de la serie 2.

\begin{tabular}{lcccc}
\hline Parámetro & Valor estimado & Error estándar & Valor de T & Valor de P \\
$\varnothing 1$ & 0.985939 & 0.0226921 & 43.4486 & 0.000000 \\
Media & 20.1855 & 17.7897 & 1.13484 & 0.261548 \\
$\delta$ & 0.283874 & & & \\
\hline
\end{tabular}

Verificación del modelo AR (1). En las Figuras 10 y 11 se observa que los residuales son ruido blanco, ya que las autocorrelaciones son significativas, por lo que el modelo elegido ajusta bien a los datos. La Figura 12 muestra el ajuste del modelo AR (1) a la serie 2. De tal forma que puede indicarse que el modelo predijo la fluctuación del número de minas de L. huidobrensis en forma satisfactoria ya que el comportamiento de la serie predicha se apega al comportamiento de la serie observada.

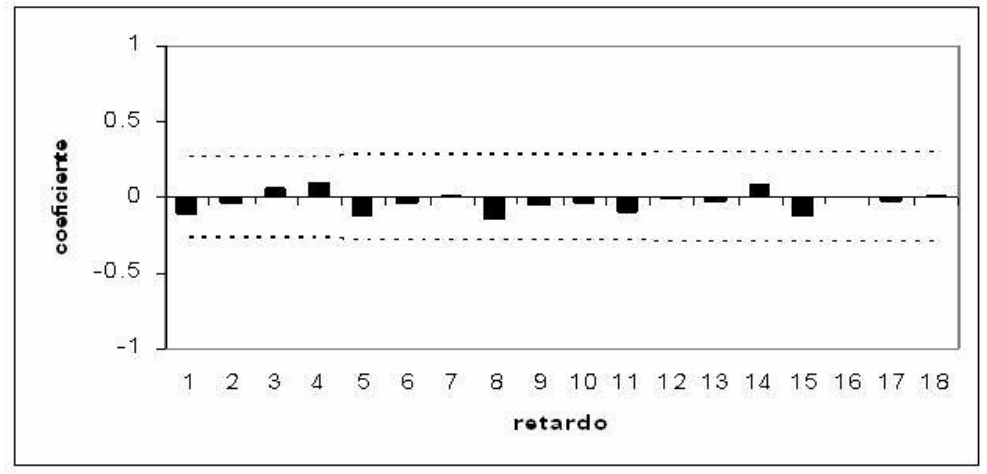

Figura 10. Función de autocorrelación (FAC) de los residuales del modelo AR(1) de la serie 2 (las líneas punteadas corresponden a \pm dos veces el valor estándar de los coeficientes). 
Hernández et al. Modelo poblacional para Liriomyza huidobrensis

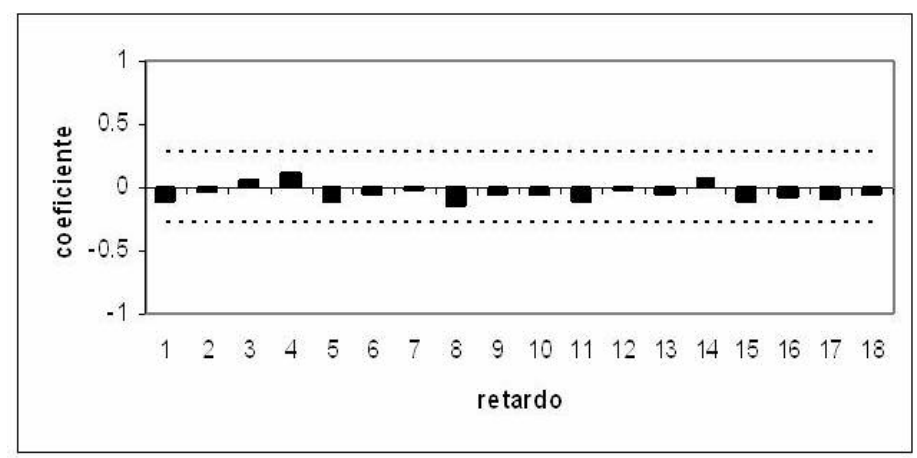

Figura 11. Función de autocorrelación parcial (FACP) de los residuales del modelo AR(1) de la serie 2 (las líneas punteadas corresponden a \pm dos veces el valor estándar de los coeficientes).

Predicción de la serie 2 con el modelo AR (1). La capacidad de predicción del modelo seleccionado fue confirmada comparando sus resultados con las seis observaciones originales restantes (56-61) de la serie 2 que se conservaron para tal fin, encontrando que los pronósticos del modelo elegido son bastante acertados en relación a los valores observados en la serie 2 (Cuadro 4; Figura 12).

Cuadro 4. Observaciones finales de la serie 2, su predicción a través del modelo AR(1) y sus límites de confianza al $95 \%$

\begin{tabular}{lcccc}
\hline Período & Serie observada & Serie predicha & Límite inferior & Límite superior \\
\hline 56 & 687 & 673.23 & 506.007 & 870.599 \\
56 & 692 & 688.473 & 458.383 & 978.265 \\
58 & 713 & 703.698 & 427.183 & 1068.94 \\
59 & 727 & 718.909 & 404.197 & 1150.83 \\
60 & 699 & 734.102 & 386.29 & 1227.06 \\
61 & 746 & 749.272 & 371.875 & 1299.23 \\
\hline
\end{tabular}

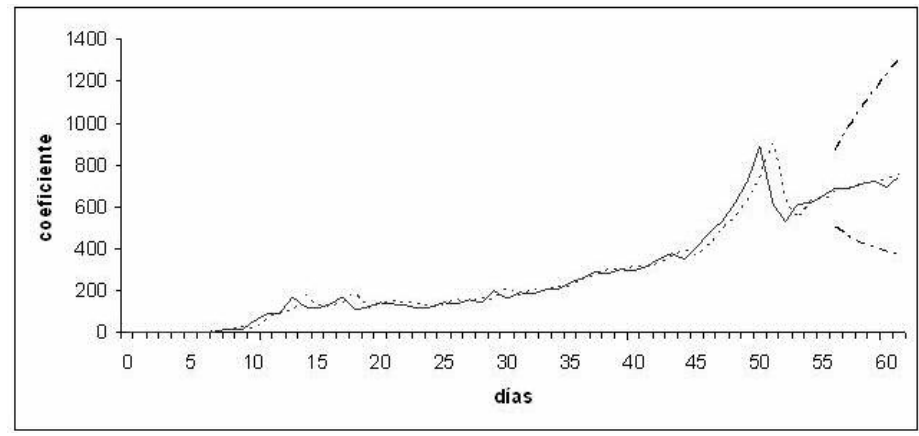

Figura 12. Ajuste del modelo $\operatorname{AR}(1)$ a la serie 2 ( _ valores observados, ....valores predichos, _. límites de confianza) 


\section{DISCUSIÓN}

La población de L. huidobrensis mostró en el desarrollo de su fluctuación poblacional un comportamiento exponencial para cada una de las series. Al inicio de cada ciclo de cultivo denota una clara tendencia a mantener su población cercana a cero en los primeros dos días después del trasplante de la plántula, posiblemente por la presencia del $100 \%$ de humedad relativa que no es apta para el desarrollo óptimo de la población del insecto, como lo mencionan en otros trabajos (Parrella, 1987; Reitz y Trumble, 2002), aunado al pequeño número de progenitores que iniciaron cada una de las poblaciones.

Posteriormente, mientras cada una de las poblaciones se establecía, paulatinamente se incremento el número de individuos de acuerdo a la cantidad de progenitores que llegaron a poblar el área de cultivo, y la capacidad reproductiva de los mismos.

La diferencia tan marcada en el número máximo de minas contadas en la serie 1 (103 minas) y la serie 2 (890 minas) se debe a la época en la que se desarrollo cada ciclo de cultivo, tendiendo a haber mayor cantidad de individuos en la condiciones más cálidas, acortándose su ciclo de vida (Lange, et al., 1957). Para la Serie 1, el banco de pupas que existía en la superficie del suelo ayudó al incremento del número de individuos que fungieron como progenitores para colonizar el cultivo. Así mismo, existen datos relacionados al hecho de que el pico máximo en la fluctuación población del género Liriomyza alcanza su máximo de crecimiento entre los meses de abril y mayo (Spencer, 1973) como se presentó en el caso de las observaciones 47 a la 55 en esta serie.

El hecho de que los resultados de modelos de series de tiempo obtenidos se hayan apegado al comportamiento natural de la población del insecto estudiado, puede servir para hacer pronósticos de su densidad poblacional que permitan tomar medidas de control oportunas y adecuadas contra esta plaga, cuando los pronósticos muestren que se rebasan los umbrales de daño establecidos,

Además, los modelos obtenidos son bastante parsimoniosos, es decir con un número de parámetros mínimo, que facilitan el cálculo de la densidad poblacional futura del insecto de referencia.

AGRADECIMIENTOS. Al Consejo Nacional de Ciencia y Tecnología (CONACyT) por el apoyo económico para la realización de esta investigación.

\section{LITERATURA CITADA}

Anuario Estadístico de la Producción Agrícola. 2005. Servicio de Información Agroalimentaria y Pesquera. Secretaría de Agricultura, Ganadería, Desarrollo Rural, Pesca y Alimentación, D.F. México. www.siap.sagarpa.gob.mx/ar-comdeanuadin.html

Box, G. E. P. \& G. M. Jenkins. 1976. Time series analysis: forecasting and control. Holden-Day. Oakland, California, U.S.A. 575 p.

Castillo M., L. E. 2005. Elementos de muestreo de poblaciones. 2da edición. Departamento de Parasitología Agrícola. Universidad Autónoma Chapingo. Chapingo, México. 267 p. 
Chandler, L. D. \& C. E. Thomas. 1983. Seasonal population trends and foliar damage of agromyzid leafminers on cantaloupe in the lower Rio Grande Valley. Journal of the Georgia Entomological Society, 18:112-120.

Foster, R. E. \& C. A. Sanchez. 1988. Effect of Liriomyza trifolii (Diptera: Agromyzidae) larval damage on growth, yield, and cosmetic quality of celery in Florida. Journal of Economic Entomology, 81: 2380-2384.

García, E. 1987. Modificaciones al sistema de clasificación climática de Koppen: para adaptarlo a las condiciones de la República Mexicana. Offset/Larios S. A. 4ta edición. México, D.F. 217 p.

Gottman, J. M. 1981. Time-series analysis: A comprehensive introduction for social scientists. Cambridge University. Cambridge. 400 p. 12

Harvir Singh, V. S. Malik \& Mukesh Kumar. 1996. Role of abiotic factors in seasonal abundance and biology of American serpentine leaf miner, Liriomyza trifolii Burgess (Diptera: Agromyzidae) on castor. Indian Journal of Ecology 23(1):34-38.

Huerta P., R. A. 2000. Diagnóstico agroecológico del cultivo de crisantemo en Texcoco, Méx. y propuestas de manejo para el control de plagas. Tesis de Maestro en Ciencias. Colegio de Postgraduados. Campus Montecillo. Montecillo, México. 170 p.

Infante G., S., M. O. Álvarez \& J. Vera G. 1983. Una aplicación de series de tiempo para poblaciones naturales de insectos. Memoria del V Congreso Nacional sobre Estadisticas Agropecuarias y Forestales. SARH. México. pp. 321-333.

Jones, P. D. \& M. Hulme. 1996. Calculating regional climatic time series for temperature and precipitation: methods and illustrations. International Journal of Climatology, 16(4):361-377.

Lange, W., A. Gricarick \& E. Carlson. 1957. Serpentine leafminer damage. California Agriculture, 11:3-5

Parrella, M.P. 1987. Biology of Liriomyza. Annual Review of Entomology, 32:201-224.

Parrella, M. P., W. W. Allen \& P. Marischita. 1981. Leafminer species cause California chrysanthemum growers new problem. California Agriculture, 35: 28-30.

Pinto, V. M., J. Vera G., D. Sotres R. \& F. Gonzalez C. 1990. Predicción de la fluctuación poblacional de Zabrotes subfasciatus Boh. (Coleoptera: Bruchidae) a través de modelos de series de tiempo. Agrociencia. Serie Matemáticas Aplicadas, Estadística y Computación, 1(1):135-157.

Redfern, M. \& Hunter M. D. 2005. Time tells: long-term patterns in the population dynamics of the yew gall midge, Toxomyia taxi (Cecidomyiidae), over 35 years. Ecological Entomology, 30(1):86-95.

Reitz, S. R. \& J.T. Trumble. 2002. Interspecific and intraspecific differences in two Liriomyza leafminer species in California. Entomologia Experimentalis et Applicata, 102: 101-113

Scargle, J. D. 1982. Studies in astronomical time series analysis. II-Statistical aspects of spectral analysis of unevenly spaced data. The Astrophysical Journal, 263: 835.

Spencer, K. 1973. Agromyzidae (Diptera) of economic importance (Series Entomologica). Springer. The Hague, Netherlands. 418 pp.

Statgraphics Plus Ver. 5.1 Professional. 2001. STSC and Statistical Graphics Corporation. Bekersville Maryland. 13

Weigend, A. S. \& N. A. Gershenfeld. 1994. Time series prediction: forecasting the future and understanding the past. Addison-Wesley. Santa Fe Institute. New, Mexico. 643 pp.

Williams, D. W. \& A. M. Liebhold. 1995. Influence of weather on the synchrony of gypsy moth (Lepidoptera: Lymantriidae) outbreaks in New England. Environmental Entomology, 24(5):987-995.

Zeger, S. L., D. Thomas, F. Dominici, J. M. Samet, J. Schwartz, D. Dockery \& A. Cohen. 2000. Exposure measurement error in time-series studies of air pollution: concepts and consequences. Environmental Health Perspectives, 108(5):419-426.

Recibido: 23 de noviembre de 2007

Aceptado: 5 de septiembre de 2008 